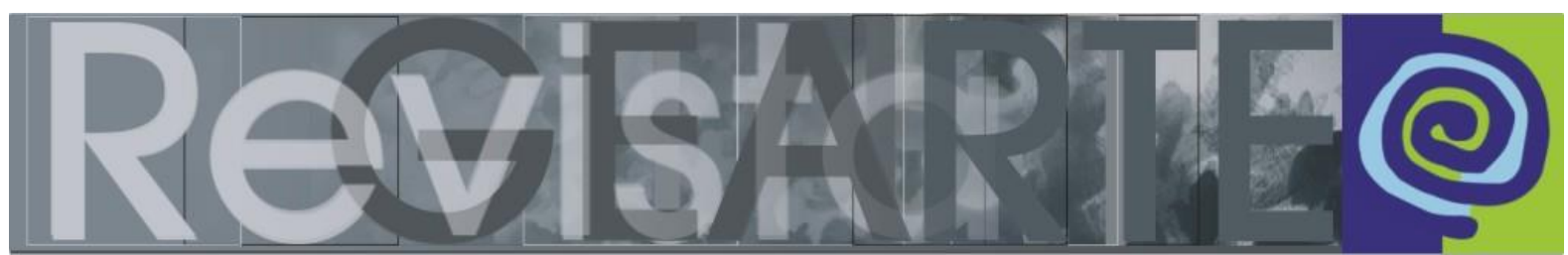

ISSN 2357-9854

\title{
Aproximación a una re-conceptualización de la educación artística en la sociedad del conocimiento en Colombia
}

Bernardo Bustamante Cardona (Universidad de Antioquia - UdeA, Medellín, Colombia)

RESUMO - Aproximação a uma reconceitualização do ensino da arte na sociedade do conhecimento na Colômbia - O texto apresenta uma análise metateórica, partindo de um problema de organização do conjunto de enfoques teóricos no campo do ensino da arte na Colômbia. Com uma abordagem metodológica cartográfica, propõe quatro vias para a compreensão do denso território que se observa. Estas vias são: a via que prioriza a história da cultura e a história da arte como fundamento para o ensino da arte; a via que vincula o ensino da arte à escola e, portanto, o inclui nas pedagogias que envolvem todos os campos do saber; a via que explica o ensino da arte desde o campo do saber; a via da epistemologia dos saberes, do saber artístico e do ensino da arte.

PALAVRAS-CHAVE

Ensino da Arte. Cartografia. Campos do saber. Colômbia.

RESUMEN - Aproximación a una re-conceptualización de la educación artística en la sociedad del conocimiento en Colombia - Se presenta un análisis metateórico, partiendo de un problema de organización del conjunto de enfoques teóricos en el campo de la Educación Artística en Colombia. Con una aproximación metodológica cartográfica, propone cuatro rutas para la comprensión del denso territorio que se observa. Estas rutas son: La ruta que prioriza la historia de la cultura y la historia del arte como fundamento para la educación artística. La ruta que articula la educación artística a la escuela y por lo tanto la incluye en las pedagogías que cubre todos los campos de saber. La ruta que explica la educación artística desde el campo de saber. La ruta desde la epistemología de los saberes, del saber artístico y la educación artística.

\section{PALABRAS CLAVE}

Educación Artística. Cartografía. Campo de saber. Colombia.

\section{Introducción}

Se trata en el artículo de realizar una aproximación metateórica, partiendo de algunos problemas planteados en la organización del panorama específico del campo de la educación artística. Cuando se analiza diversos materiales se presentan interrogantes como los siguientes: ¿cómo encontrar un orden interno de las diversas fuentes de fundamentación de la educación artística?, ¿cómo inducir un orden posible de las diversas posturas?, por lo tanto ¿cómo encontrar una clarificación del panorama de las teorías sobre la educación artística, hoy presentadas en un territorio diverso, discontinuo y desordenado? 
La idea expuesta aspira a indicar la posibilidad de encontrar un orden en los postulados de diferentes autores, por lo tanto, permite presentar una hipótesis de organización de los fundamentos de la educación artística en cuatro rutas posibles, entre otras, a saber:

- la ruta que prioriza la historia de la cultura y la historia del arte como fundamento para la educación artística;

- la ruta que articula la educación artística a la escuela y por lo tanto la incluye en las pedagogías que cubren todos los campos de saber;

- la que explica la educación artística desde el campo de saber, dentro y fuera de la escuela;

- la ruta desde la epistemología de los saberes, del saber artístico y la educación artística.

Esta iniciativa está enmarcada en la lectura de la condición actual del desarrollo que consideramos sea la sociedad del conocimiento, con respecto a esta podemos citar a Manuel Castells (2013) el cual nos propone un análisis de la sociedad actual desde un cambio en la base productiva y en la infraestructura que lo soporta.

Si para el siglo XX se impone la industria del acero y del carbón y se marca una transformación esencial en la fabricación de objetos, este fenómeno se presenta aunado con la mecanización de la producción, la organización fordista del trabajo en cadena y la ingeniería que la soporta. Estas sociedades encuentran sus límites hacia el final del siglo XX y en este tiempo se produce un cambio extraordinario en las condiciones de producción, la introducción a gran escala del valor de la información, la computadora como intermediaria, como también el advenimiento de la red como infraestructura global de la nueva economía, por esto, se puede entender que se presenta el cambio en la base de producción que modela una sociedad del conocimiento.

\section{Método}

Para lograr el objetivo propuesto en este artículo se hace una revisión de fuentes teóricas, nacionales e internacionales, relacionadas con la educación artística 
en particular; se realiza luego una selección de artículos de diversos docentes e investigadores colombianos y se inicia un camino metateórico, a través de una cartografía, partiendo de la hipótesis del autor, la cual propone que pueden trazarse unas rutas que permita establecer parámetros de recorrido y de conceptualización de la Educación Artística y luego señalar la emergencia de nuevas rutas en las sociedades contemporáneas o sociedades de conocimiento.

La cartografía nos conduce a extender el panorama del universo de la investigación en topografías diversas y en temporalidades diferentes, pero también nos induce a reconocer las rutas posibles y a llevar a cabo el análisis metateórico que permite organizar las diversas teorías que se presentan. En cuanto a cartografiar nos dice Helena Tatay (2012) como comisaria de la exposición "Cartografías contemporáneas. Dibujando el pensamiento":

El ser humano cartografía el mundo en un intento de captar la realidad en que
vive. Desde siempre, los mapas han representado, traducido y codificado
todo tipo de territorios físicos, mentales y emocionales. La representación del
mundo ha evolucionado en los últimos siglos y, ahora, con la globalización e
Internet, se han transformado definitivamente los conceptos tradicionales de
tiempo y espacio, así como los métodos de representación del mundo y el
conocimiento. Con este cambio de paradigma, los artistas contemporáneos
se cuestionan los sistemas de representación y ofrecen nuevas fórmulas para
clasificar la realidad. Cartografías contemporáneas. Dibujando el
pensamiento propone un mapa de las cartografías elaboradas por artistas de
los siglos XX y XXI, con el objetivo último de invitar a los visitantes a
interrogarse sobre los sistemas de representación que usamos y las nociones
subyacentes. (TATAY, 2012, p. 1)

\section{Antecedentes}

Desde hace algunos años nos hemos dado a la tarea de recopilar y clasificar los diversos puntos de vista que manifiestan los expertos en educación artista, tanto en Colombia como en América Latina, también en Estados Unidos y la Unión Europea incluyendo España. La propuesta en un inicio estaba dirigida a proporcionar argumentos válidos a la investigación y a los programas de formación de docentes en el campo de saber específico.

Al abordar este panorama, que se presenta tan diverso, se propuso una serie de distinciones posibles, entre ellas el hacer diferenciaciones por el país de origen; también hacer clasificaciones según el año de publicación de los textos; se pensó 
además extender la agrupación temática presentada por Mejía (2009). Estas alternativas, se concluyó, no permitían ver el panorama de la educación artística contemporánea en sus diversas aristas y complejidad, o presentan saltos categoriales. Por lo tanto se emprendió la búsqueda de algunos criterios que debían surgir de una mirada metodológica diferente y se optó por la cartografía como método.

Presentamos aquí cuatro rutas posibles en el panorama del análisis de los discursos sobre la educación artística. Bajo esta mirada, se propondrá posteriormente el estudio de las condiciones de surgimiento de algunos de ellos y, se espera encontrar rutas pertinentes para la sociedad del conocimiento que aún están poco exploradas.

\section{Las rutas}

Las cuatro rutas son derivadas de una organización posible sobre el material recopilado, textos físicos y virtuales, artículos y capítulos de libros que podrían denominarse el universo de la investigación, y este universo se divide en unidades de análisis específicas. Pero además se propone una mirada sobre el territorio, y así permitir la compresión del problema teórico; teniendo lo anterior, se puede comprender la coexistencia de diversos puntos de vista y de sus múltiples relaciones rizomáticas, como también nos permite distinguir algunas posibles rutas emergentes.

\section{La ruta que prioriza la historia de la cultura y la historia del arte}

En esta primera ruta los autores desarrollan un análisis que hace énfasis en la influencia del arte, de sus movimientos intrínsecos, además de los postulados de la cultura en la educación artística. La nombramos La ruta de la historia de la cultura y el arte. En esta ruta se encuentra como principio de orden, como característica, el hecho de priorizar las transformaciones ocurridas en el acontecer del arte, haciendo de los postulados del arte el fundamento de la educación. Por ejemplo si se ha producido una ruptura entre la modernidad y la posmodernidad en el arte, esta se refleja en la educación y se presenta la propuesta de la educación posmoderna. Esta idea es clara en la tesis de Efland, Freedman y Stuhr (2003) con su texto "La educación en el arte posmoderno". 
En el panorama colombiano se encuentra Olga Olaya (2008) que piensa la educación artística sobre estas transformaciones artísticas y culturales. En su texto presentado en el Seminario Internacional de Educación Artística en Chile, "Consideraciones y recomendaciones para el desarrollo de una Educación Artística", Olaya le apuesta a un orden como el siguiente para las concepciones o tendencias en educación artística:

Concepción instruccional para el caso de las disciplinas que reproducen aprendizajes en torno a sus normas, proporciones y cánones, validados en diferentes contextos internacionales, (...) Sus contenidos tuvieron extensa incidencia y permanencia en los currículos colombianos. (OLAYA, 2008, p. 23)

Concepción autoexpresiva en la cual se potencia la sensibilidad, la capacidad creadora, y el respeto por la emoción y el sentimiento, privilegiando la práctica del estudiante como expresión libre o autoexpresión (...). (OLAYA, 2008, p. 24)

Concepción funcional que se refiere a aquel tipo de educación que se ha implementado en los grados superiores, (...) acercando al estudiante a las primeras nociones sobre diseño de interiores, diseño gráfico y en algunos casos diseño industrial. (OLAYA, 2008, p. 24)

Concepción interdisciplinar que pone de manifiesto la incursión de las "ciencias del arte" como la estética, la crítica o la historia del arte a la par con la creación artística del lenguaje disciplinar (música, danza, teatro, artes plásticas, diseño audiovisual, entre otras). (OLAYA, 2008, p. 24)

Nuevas concepciones del siglo XXI se presentan como unas propuestas para el docente quien, para responder a las necesidades y exigencias del mundo contemporáneo, tiene múltiples opciones para escoger y asumir posiciones conscientes y fundamentadas desde propuestas, pensamientos y autores, para su ejercicio. (OLAYA, 2008, p. 25)

El argumento central es la condición de las artes para dar el fundamento a la educación artística y cultural, argumento válido, que responde a un análisis del saber pedagógico influenciado por el arte, pero también, presupone, algunas veces, el principio basado en que el arte conlleva su propia pedagogía y no tiene necesidad de atender a la articulación con otros saberes para su enseñabilidad. Desarrolla a profundidad la articulación que tiene la educación artística con las prácticas artísticas contemporáneas. 
En este primer tamizaje sobre la literatura de la educación artística también encontramos otro enfoque que nos indica el trayecto de la escuela y su relación con la pedagogía tradicional.

\section{La ruta que articula la educación artística a la escuela y por lo tanto la incluye en las pedagogías que cubren todos los campos de saber}

Un segundo enfoque lo nombramos El trayecto que articula la escuela y la pedagogía en todos sus campos de saber. Se hace énfasis en este enfoque en la pertinencia de la pedagogía como lugar de todo análisis de enseñanza, por lo que es la pedagogía en su desarrollo, llámese ciencia o disciplina, la que combina sus elementos y herramientas de análisis para apoyar a las artes en la posibilidad de la enseñabilidad, por lo tanto deberá ser la estructura y el devenir de la pedagogía la que aporte las luces de las prácticas en la escuela. Este análisis se puede ver en la postura pedagógica de los documentos estatales, como son las Orientaciones Pedagógicas para la Educación Artística en Básica y Media (2010) ofrecidas por el Estado Colombiano, en ellas se postula un enfoque por competencias como la dirección oficial de la educación artística. Las Orientaciones define la Educación Artística así:

(...) se trata de una educación por las artes, que busca contribuir a la formación integral de los individuos a partir del aporte que realizan las competencias específicas sensibilidad, apreciación estética y comunicación al desarrollo de las competencias básicas (...) (MINISTERIO DE EDUCACIÓN COLOMBIANO, 2010, p. 17).

Qué son las competencias, está en el ámbito del discurso pedagógico de la escuela colombiana, este concepto no proviene de las artes o de las prácticas culturales, es un enfoque sobre el saber en las prácticas escolarizables. Las competencias son un enfoque estatal que condiciona la educabilidad y la enseñabilidad y los sitúa en el contexto de la sociedad contemporánea y el proyecto productivo. Para comprender que es competencia en el contexto colombiano nos referimos a las Orientaciones. Éste documento dice:

En resumen, a partir de los diferentes aportes realizados al deslinde de la noción de competencia se puede decir que ésta se refiere a la habilidad del individuo para responder con diferentes grados de efectividad a un problema de la realidad, poniendo en movimiento diferentes recursos cognitivos, no cognitivos y del entorno. "La competencia involucra el uso de conocimientos conceptuales, procedimentales y actitudinales. Asimismo, articula capacidades innatas (como la de adquirir un 
lenguaje) y adquiridas (como los conocimientos)" (BOLÍVAR; PEREYRA, 2006, p. 7). (MINISTERIO DE EDUCACIÓN COLOMBIANO, 2010, p. 24)

Por lo tanto el concepto de competencias es externo al saber artístico, en éste no se ha establecido el concepto y su pertinencia, pero es propio del saber pedagógico, y por lo tanto, condiciona el sentido de la educación artística como práctica en la escuela básica regular; ahora bien esto no quiere decir que la propuesta del Ministerio de Educación Colombiano, con respecto a la educación artística, sea apropiada al saber artístico y sus prácticas profesionales, como tampoco determina la apropiación de experiencias indispensables en el arte contemporáneo como las que Gadamer (1991), en su texto la Actualidad de lo Bello, dice: "¿Cuál es la base antropológica de nuestra experiencia del arte? Esta pregunta tiene que desarrollarse en los conceptos de "juego, símbolo y fiesta"' (GADAMER, 1991, p. 31).

Las competencias no nos llevan a pensar en el ambiente de la lúdica o el juego o la fiesta en la educación artística, ¿será que la necesidad de producción requiere hacer indebidos ajustes y corsés no pertinentes para el área de conocimiento? ¿Sera que el Estado Colombiano está tratando de colocar todas las prácticas de la escuela en el mismo conjunto sin identificar sus diferencias, con el afán de dar cuenta de una política y no de una postura epistemológica o pedagógica pertinente para cada área de saber?

\section{La ruta que explica la educación artística desde el campo de saber, dentro y fuera de la escuela}

La tercera ruta la nombramos la ruta que explica la educación artística desde el campo de saber, dentro y fuera de la escuela; es una mirada que posa su atención en el hecho, empírico por lo demás, de la presencia de la educación artística en la escuela, como también en la en educación informal y en diversas prácticas sociales. Por lo tanto, en este enfoque, la educación artística no se reduce al concepto de pedagogía tradicional sino al desarrollo de un campo en crecimiento. En este sentido se encuentran los análisis de Bernardo Bustamante Cardona (2012) en textos como "Aportes de los encuentros de experiencias significativas al campo de la educación artística": 
En una sociedad en conflicto armado (...) se debe preguntar cuáles son los aportes de la Educación Artística. Pero esta pregunta no debe ser enfocada a la acción puntual en el aula de clase, debe abarcar el sistema social y el sistema de intercambios simbólicos y culturales. Los Encuentros de Experiencias Significativas han propiciado la estructuración, la comprensión, la definición y la expansión de un campo de saber que, en su totalidad, emerge en el sistema social como un espacio de debate y de tensiones que aporta a los cambios socioculturales requeridos en el momento histórico. (BUSTAMANTE CARDONA, 2012, p. 226)

Entendiendo el concepto de campo desde el sentido que le ha dado Bourdieu, como un conjunto de elementos en tensiones y relaciones, se puede decir que el campo de la Educación Artística está en construcción y aporta desde su misma estructuración en el desarrollo de las investigaciones, prácticas y teorías entre otros componentes de la Educación Artística y Cultural en Colombia. En el texto Análisis Prospectivo de la Educación Artística en Colombia al horizonte del 2019, presentado por el Ministerio de Cultura (2006), se establece este punto de vista:

La cuarta variable es el reconcomiendo de la educación artística como campo de conocimiento, y es sin duda la más compleja de todas. A través de esta variable se ve una valoración de la educación artística más elevada en el futuro, basada en la afirmación de las particularidades y la esencia del pensamiento y la práctica artísticos. (MINISTERIO DE CULTURA, 2006, p. 34)

Por lo tanto la ruta que integra el concepto de campo de saber, todavía es reciente y está por explorarse y desarrollarse en Colombia, tiene valor por considerar los diversos elementos del campo como son: los investigadores, las publicaciones, las tecnologías, las leyes, las prácticas, las instituciones, los eventos, y además permitir un acercamiento al análisis del sistema educativo y la relación con los subsistemas.

\section{La ruta desde la epistemología de los saberes, del saber artístico y educación artística}

La cuarta ruta la nombramos La ruta desde la epistemología de los saberes, del saber artístico y educación artística. Esta ruta está muy poco explorada, pero es pertinente para dar a entender los cambios que ocurren en los saberes, en este caso atiende el devenir intrínseco del saber pedagógico y del arte desde el punto de vista de la organización conceptual y el método, haciendo énfasis en la pregunta por la posibilidad de un saber, de sus condiciones de formación y sobre todo de la condición paradigmática que define. 
Entendemos el sentido de la ruta epistemológica cuando los discursos del saber científico social y de la pedagogía están cruzados por rupturas o por dis-continuidades en el plano de la estructuración de los discursos.

El enfoque epistemológico pretende acercar el análisis de la educación artística a los procesos teóricos y de investigación de otras ciencias sociales, por lo tanto encuentra en común que las ciencias sociales han mostrado diversas epistemes como la positivista, la estructural, la pos estructural, la sistémica y la compleja.

En este sentido son pocos los análisis que se encuentran en Colombia sobre la ruta epistemológica en este campo teórico, y se espera por lo tanto una emergencia en esta ruta que permita cualificar los aspectos de interdisciplinariedad de la educación artística y de la articulación de las diferentes rutas, en un panorama complejo.

\section{Conclusiones}

El análisis propuesto conduce a referirnos al panorama teórico de la Educación Artística en especial en Colombia, partiendo de una aproximación sobre el territorio de las teorías expuestas en varias publicaciones, este panorama muestra una realidad poco ordenada, se podría decir caótica y con diferenciaciones en varios planos del conocimiento e indiferenciado en muchas otros. Lo que se postula como hipótesis en este artículo, es la posibilidad de establecer algunas rutas, entrar en el panorama de la meta-teoría para postular un sentido con diferenciación progresiva y reconciliación integradora, como propone Novak.

Para este trabajo se propone una iniciativa cartográfica que permite afrontar los problemas de la formalización del discurso de la educación artística y los fundamentos que la soportan. El proceso conduce a proponer, como hipótesis inicial de trabajo, varias rutas entre las que se encuentran: la ruta de la historia de la cultura y el arte, el camino que articula la escuela y la pedagogía en todos sus campos de saber, la ruta desde las prácticas de la educación artística dentro y fuera de la escuela y la ruta desde la epistemología de los saberes. En el análisis se encuentra que la ruta de 
menor presencia en los marcos teóricos de la E. A. es la del enfoque epistemológico, siendo que, en la actualidad es de interés para las ciencias sociales, ya que con este enfoque se puede relacionar las investigaciones en educación artística con el desarrollo de las ciencias sociales, y a través de este camino, encontrar sentido a los métodos y pensamientos de la complejidad y de las ciencias del caos, que expuesto por Morín y Luhmann, permiten consolidar una investigaciones de tipo cualitativo y vislumbrar las transformaciones de tipo metodológico en el campo de la Educación Artística en Colombia y América Latina.

\section{Referencias}

BALLESTER, Luis; COLOM, Antoni J. Epistemología de las ciencias sociales. Valencia: Tirant Humanidades, 2012.

BUSTAMANTE CARDONA, Bernardo. Aportes de los encuentros de experiencias significativas al campo de la Educación Artística. Revista Colombia Praxis \& Saber, Tunja, v.3, n. 6, p. 213-228, 2012. Disponible en: <http://revistas.uptc.edu.co/revistas/index.php/praxis_saber/article/view/2010/2005>. Acceso el: 3 abr. 2016.

CASTELLS, Manuel; CARAÇA, Joao; CARDOSO, Gustavo. Después de la crisis. Barcelona: Alianza Editorial, 2013.

EFLAND, Arthur; FREEDMAN, Kerry; STUHR, Patricia. La educación en el arte posmoderno. Barcelona: Ed. Paidós, 2003.

GADAMER, Hans Georg. La actualidad de lo bello. México: Ed. Paidós, 1991.

MEJÍA, Silvana; YARSA, Alexander. Discursos y conceptualizaciones sobre Educación Artística en Revistas Colombianas. Educación y Pedagogía. Medellín. Universidad de Antioquia. Facultad de Educación. v. 2. Fascículo 55. p. 171-188. Septiembre/ diciembre, 2009.

MINISTERIO DE EDUCACIÓN COLOMBIANO. Orientaciones Pedagógicas para la Educación Artística en Básica y Media. Documento n. 16. Bogotá: Ministerio de Educación, 2010.

MINISTERIO DE CULTURA. Análisis prospectivo de la Educación Artística colombiana 2006-2019. Documento oficial. Bogotá: Ministerio de Cultura, 2006.

OLAYA PARRA, Olga Lucia. Consideraciones y recomendaciones para el desarrollo de una Educación Artística. Seminario Internacional de Educación Artística en Chile. Programa Educación Artística en la Formación Superior Inicial y Continua. Santiago de Chile: Ed. Consejo Nacional de la Cultura y las Artes, 2008. Disponible en: <http://www.estaciondelasartes.com/wp-content/uploads/gravity_forms/267af245c3a56c245328a35ecd40f1 bac/2012/10/Libro-Seminario-Internacional-de-Educaci\%C3\%B3nartistica2008.pdf>. Acceso el: 12 maio 2016.

TATAY, Helena. Cartografías contemporáneas. Dibujando el pensamiento. Metalocus. Revista digital. 2012. Disponible en: <http://www.metalocus.es/es/historic?keys=cartografias\%20> y $<$ http://prensa.lacaixa.es/obrasocial/exposicion-cartografias-contemporaneas-caixaforum-barcelonaesp_816-c-16501_.html>. Acceso el: 5 mar. 2016. 


\section{Bernardo Bustamante Cardona}

Filósofo, artista y docente de la Universidad de Antioquia, en Medellín (Colombia). Maestro en Educación énfasis en cognición y creatividad por la Pontificia Universidad Javeriana. Docente de intercambio con la École Superieure d'Art de Lorient, Francia; Universidad Industrial de Santander; Universidade Federal de Minas Gerais, Brasil.

E-mail: berbus00@hotmail.com

Currículo:

http://scienti.colciencias.gov.co:8081/cvlac/visualizador/generarCurriculoCv.do?cod_rh=0000564419

Recebido em 17 de maio de 2016 Aceito em 21 de junho de 2016 